\title{
CONF-960401-16 \\ INACCURACIES IN SNEDDON'S SOLUTION FOR ELASTIC INDENTATION BY A RIGID CONE AND THEIR IMPLICATIONS FOR NANOINDENTATION DATA ANALYSIS
}

\author{
A. BOLSHAKOV* and G.M. PHARR* \\ * Department of Materials Science, Rice University, 6100 Main St., Houston, TX 77005
}

\section{ABSTRACT}

Methods currently used for analyzing nanoindentation load-displacement data to determine a material's hardness and elastic modulus are based on Sneddon's solution for the indentation of an elastic half-space by a rigid axisymmetric indenter. Although this solution is widely used, no attempts have been made to determine how well it works for conditions of finite deformation, as is the case in most nanoindentation experiments with sharp indenters. Analytical and finite element results are presented which show that corrections to Sneddon's solution are needed if it is to be accurately applied to the case of deformation by a rigid cone. Failure to make the corrections results in an underestimation of the load and contact stiffness and an overestimation of the elastic modulus, with the magnitude of the errors depending on the angle of the indenter and Poisson's ratio of the half-space. For a rigid conical indenter with a half-included tip angle of $70.3^{\circ}$, i.e., the angle giving the same area-to-depth ratio as the Berkovich indenter used commonly in nanoindentation experiments, the underestimation of the load and contact stiffness and overestimation of the elastic modulus may be as large as $13 \%$. It is shown that a simple first order correction can be achieved by redefining the effective angle of the indenter in terms of the elastic constants. Implications for the interpretation of nanoindentation data are discussed.

\section{INTRODUCTION}

This paper deals with Sneddon's solution for the indentation of an elastic half-space by a rigid axisymmetric indenter [1,2]. Sneddon's solution is the comerstone for determining elastic moduli from nanoindentation load-displacement data $[3,4]$, and as such, the accuracy with which the elastic moduli can be measured depends on how well Sneddon's solution describes real material behavior.

The primary result of Sneddon's work of importance in this paper is the relation [3]:

$$
E_{\text {eff }}=\frac{E}{1-v^{2}}=\frac{\sqrt{\pi}}{2} \frac{S}{\sqrt{A}}
$$

Here, $E_{\text {eff }}$ is the effective elastic modulus defined in terms of Young's modulus $E$ and Poisson's ratio $\mathrm{V}, \mathrm{S}$ is the contact stiffness, and $\mathrm{A}$ is the projected contact area. The equation applies to any rigid axisymmetric indenter, including singular indenters such as cones [3]. Since all of the quantities on the right hand side can be either measured or estimated from indentation loaddisplacement data, Eqn.1 forms the basis of elastic modulus measurement by load and displacement sensing indentation methods [3-5].

One potential problem in using Sneddon's solution is that it is derived for conditions of small deformation. These conditions are often well-satisfied for shallow indentations made by blunt indenters such as a sphere, but for sharp indenters like the Berkovich triangular pyramid used commonly in nanoindentation work, the small-deformation formulation may be inaccurate. An indication that there may be a problem for sharp indenters was recently encountered in finite element simulations of elastic/plastic deformation by a rigid cone using a finite element code which accounts for finite strain and rotation [6]. No matter how carefully the mesh size and shape were controlled, the elastic modulus derived from the simulated indentation loaddisplacement curves using Eqn.1 was always 10-20\% larger than that used as input in the simulation. A similar result has been reported by Ritter et al [7]. Larsen and Simo [8], on the

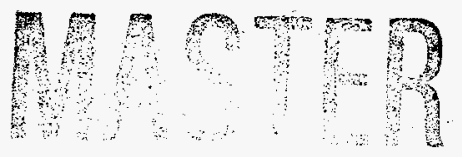

-The submitted manuscript has been authored by a

The submitted manuscript has bernent under contract No. contractor of the U. S. Governordingly, the U.S.

DE-AC05-960R22464. Accordingly, the U.S.

Government retains a nonexclusive, royally of this

to publish or reproduce the published form of 
other hand, found relatively good agreement between the input and derived elastic modulus in their finite element simulations, but in carefully re-assessing their results, we have found that the procedure they used to measure the contact stiffness significantly underestimated its actual value. When corrected, the discrepancy between the input and derived elastic modulus in their finite element studies is even greater than in ours.

The discrepancies in the finite element results just described led us to undertake a careful study of the problems which can occur in applying Sneddon's equations to sharp indenters. Here, we report some observations for elastic indentation by a rigid cone and discuss their implications for the measurement of elastic modulus by nanoindentation methods. A more complete report, including implications for elastic/plastic deformation and the measurement of contact area and hardness, will be presented in a subsequent report [9].

\section{INACCURRACIES IN SNEDDON'S SOLUTION FOR A RIGID CONE}

The problem in applying Sneddon's solution to deformation by sharp indenters can be illustrated by considering his analysis of the indentation of an elastic half-space by a rigid cone. For a cylindrical coordinate system with a free surface initially at $z=0$, the boundary conditions used by Sneddon are:

$$
\begin{aligned}
& \left.\sigma_{z z}\right|_{z=0}=0, \quad r>a \\
& \left.\sigma_{r}\right|_{z=0}=0, \quad r \geq 0 \\
& \left.w\right|_{z=0}=D-\cot (\phi) r, \quad r<a .
\end{aligned}
$$

Here, $\mathrm{a}$ is the contact radius, $\mathrm{D}$ is the depth of penetration, $\phi$ is the half-included angle of the cone, $w$ is the vertical displacement of the surface, and the $\sigma_{i j}$ are the components of the stress tensor. The first two boundary conditions specify the tractions on the $\mathrm{z}=0$ surface (no friction is assumed), and the third condition forces the vertical displacements of the surface inside the circle of contact to be consistent with the geometry of the cone. Posed in this manner, the problem can be solved by the method of Hankel transforms [1,2]. The primary result of importance in this

discussion is the expression for the radial displacements, $u$, of points inside the circle of contact, which is given by:

$$
\left.u\right|_{z=0}(r, \phi)=\frac{(1-2 v)}{4(1-v)} \cot (\phi) r\left[\ln \frac{r / a}{1+\sqrt{1-(r / a)^{2}}}-\frac{1-\sqrt{1-(r / a)^{2}}}{(r / a)^{2}}\right], \quad r<a
$$

Close examination of this equation shows that the surface radial displacements vanish only when Poisson's ratio $v$ is 0.5 , i.e, the material is incompressible, or when $\phi=90^{\circ}$. Thus, during indentation by a sharp cone, the surface radial displacements for most materials of practical interest are finite. An important consequence is that when the radial displacements are taken into account, the deformed surface inside the area of contact is not conical but rather described by (see [9] for details):

$$
r=\frac{z}{\cot (\phi)}+\frac{(1-2 v)}{4(1-v)} z\left[\ln \frac{\mathrm{z} /[\mathrm{a} \cot (\phi)]}{1+\sqrt{1-\mathrm{z}^{2} /[\mathrm{a} \cot (\phi)]^{2}}}-\frac{1-\sqrt{1-\mathrm{z}^{2} /[\operatorname{acot}(\phi)]^{2}}}{\mathrm{z}^{2} /[\operatorname{acot}(\phi)]^{2}}\right] .
$$

Based on the predictions of Eqn. 6, the shapes of surfaces deformed by a $70.3^{\circ}$ cone for materials with various values of Poisson's ratio are shown in Figure 1 . Note that only in the case of $v=0.5$ is the deformed surface consistent with the $70.3^{\circ}$ conical geometry; that is, for all other Poisson's ratios, the surface is displaced inward from the $70.3^{\circ}$ cone and is slightly curved. 


\section{DISCLAIMER}

Portions of this document may be illegible in electronic image products. Images are produced from the best available original document. 



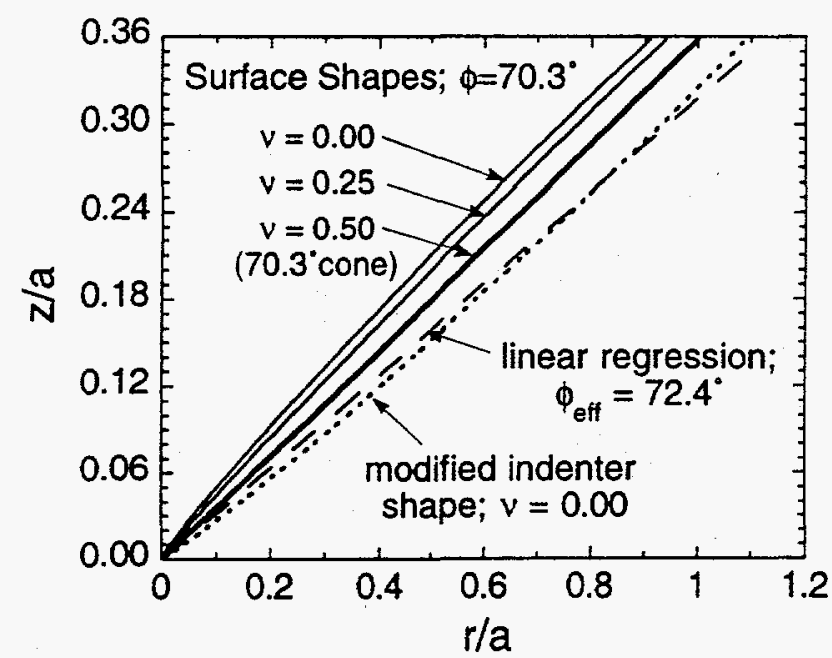

Fig. 1. The shapes of surfaces indented by a $70.3^{\circ}$ cone when the radial displacements in Sneddon's solution are included. Also shown is the shape of the alternative indenter needed to account for the radial displacements for the case of $v=0$, and the linear approximation to the shape which defines the effective cone angle, $\phi_{\text {eff }}$.

What this means is that in most cases of practical interest, Sneddon's solution applies not to a perfect cone, but to a cusped shaped indenter which approximates to a cone. As we will show in the next sections, this small deviation has important consequences for the measurement of elastic moduli by nanoindentation methods.

\section{MODIFICATIONS TO THE SOLUTION}

A solution which more accurately describes the indentation of an elastic material by a rigid cone rather than a cusp-shaped indenter can be achieved by modifying the boundary condition in Eqn. 4 to account for the finite radial displacements of points along the surface of contact. Under these circumstances, a more appropriate statement of the boundary condition is:

$$
\left.\mathrm{w}\right|_{\mathrm{z}=0}=\mathrm{D}-\cot (\phi)[\mathrm{r}+\mathrm{u}(\mathrm{r})], \quad \mathrm{r}<\mathrm{a} .
$$

This boundary condition assures that the geometry of the deformed surface will be conical with a half-included angle $\phi$ which matches that of the indenter.

Two different approaches may be used to solve this problem - one exact and one approximate. The exact solution makes use of an indenter which deviates from the conical geometry, chosen so that the deformed surface will be a perfect cone of angle $\phi$ when the radial displacements are taken into account. The solution is obtained by treating the function $u(r)$ in Eqn. 7 as an unknown and deriving an expression for $\mathrm{u}(\mathrm{r})$ using relations developed by Sneddon for the radial displacements of a general axisymmetric indenter [2]. Normalizing all length dimensions with respect to the contact radius, a, the approach leads to non-dimensional radial displacements given by:

$$
\begin{array}{r}
\overrightarrow{\mathrm{u}}(\rho)=\frac{(1-2 v)}{2(1-v)} \frac{\cot (\phi)}{\pi}\left[\frac{\pi}{4} \rho \ln \frac{\rho}{1+\sqrt{1-\rho^{2}}}-\frac{\pi}{4} \frac{1-\sqrt{1-\rho^{2}}}{\rho}+\frac{1}{\rho} \int_{0}^{1} \frac{\mathrm{y} \overline{\mathrm{u}}(\mathrm{y})}{\sqrt{1-\mathrm{y}^{2}}} \mathrm{dy}-\right. \\
\left.\frac{1}{\rho} \int_{\rho}^{1} \frac{\mathrm{x}^{2}}{\sqrt{\mathrm{x}^{2}-\rho^{2}}} \mathrm{dx} \int_{0}^{x} \frac{\overline{\mathrm{u}}^{\prime}(\mathrm{y})}{\sqrt{\mathrm{x}^{2}-\mathrm{y}^{2}}} \mathrm{dy}\right],
\end{array}
$$

where $\rho=r / a$ and $\bar{u}(\rho)=u(r) / a$. Numerical techniques can be used to solve for $u(r)$, which can be used in combination with other results derived by Sneddon to provide an exact solution [2]. However, because the solution requires numerical evaluation, it is somewhat cumbersome and awkward. As a result, we limit the discussion here to the second solution technique which has the distinct advantage of having a very simple, closed form. The solution is only approximate, 
but may prove to be more practical in nanoindentation data analysis. The accuracy of the solution will be checked by comparison to finite element simulations.

In the approximate solution, the alternative indenter is constructed by taking the perfect conical geometry with half-included angle $\phi$ and increasing the radius at each point along its surface by an amount equal to the magnitude of the radial displacements in Eqn. 5 . The shape of the indenter constructed in this manner for the case of $v=0$ is shown in Figure 1. The rationale is that since the radial displacements of the surface in Sneddon's solution are negative, increasing the radius of the perfect cone by an amount equal to the expected radial displacements should, to a first approximation, produce a deformed surface having a geometry close to the intended conical shape. A second assumption made in the analysis is that the curvature of the surface of the modified indenter is small enough to be ignored. In this case, the modified indenter can be modelled as a cone with an effective included angle, $\phi_{\text {eff }}$, slightly greater than that of the ideal cone (see Figure 1). Linear regression of the shape of the modified indenter yields:

$$
\cot \left(\phi_{\text {eff }}\right)=\frac{\cot (\phi)}{\beta}
$$

where the parameter $\beta$ is given by:

$$
\beta=1+\frac{(1-2 v)}{4(1-v)}\left(3-\frac{\pi}{2}\right) \cot (\phi) .
$$

Eqn. 9 shows that the effective indenter angle, $\phi_{\text {eff, }}$, depends on the cone angle, $\phi$, and the Poisson's ratio of the material. In the limit of incompressible deformation $(v=0.5), \phi_{\text {eff }}$ is exactly equal to $\phi$, but when $v$ is small, the two angles can be significantly different. For $v=0$ and $\phi=70.3^{\circ}$, i.e., for a cone with the same area-to-depth ratio as the Berkovich indenter, the effective cone angle is $\phi_{\text {eff }}=72.4^{\circ}$. The attractiveness of this approach is that all of the relations derived by Sneddon for the indentation of an elastic half-space by a rigid cone still apply provided the indenter angle $\phi$ is replaced by $\phi_{\text {eff. }}$ Thus, the load-displacement relation is:

$$
P=\frac{2}{\pi} \frac{E}{1-v^{2}} D^{2} \tan \left(\phi_{\text {eff }}\right)=\beta \frac{2}{\pi} \frac{E}{1-v^{2}} D^{2} \tan (\phi)
$$

and the modified form of Eqn. 1 is:

$$
E_{\text {eff }}=\frac{E}{1-v^{2}}=\frac{1}{\beta} \frac{\sqrt{\pi}}{2} \frac{S}{\sqrt{A}}
$$

The extent to which the modified relations deviate from Sneddon's original formulation depends on the magnitude of the parameter $\beta$, which in turn depends on both Poisson's ratio and the cone angle. Limiting the discussion here to $\phi=70.3^{\circ}$, the largest deviations from Sneddon's original solutions occur when $v=0$, for which $\beta=1.13$. Thus, for materials with small Poisson's ratios, Sneddon's solution can underestimate the load and overestimate the elastic modulus by as much as $13 \%$. As previously discussed, an overestimation of the modulus is exactly what has been observed in finite element simulations. For a more typical value of $v$ like $0.25, \beta=1.09$, so a $9 \%$ overestimation is expected in the modulus. When $v=0.5, \beta=1.00$, and Sneddon's original equations are accurate.

\section{COMPARISON TO FINITE ELEMENT SIMULATIONS}

To check on the applicability and accuracy of the approximate solution, finite element simulations were conducted using the ABAQUS finite element code. The indenter was modeled as a rigid cone with a half-included angle of $70.3^{\circ}$ and the material as isotropic elastic with Young's modulus $E$ and Poisson's ratio $v$. All simulations were performed to the same indentation depth, $\mathrm{D}=100 \mathrm{~nm}$, using a finite element mesh similar to one used previously [6]. 


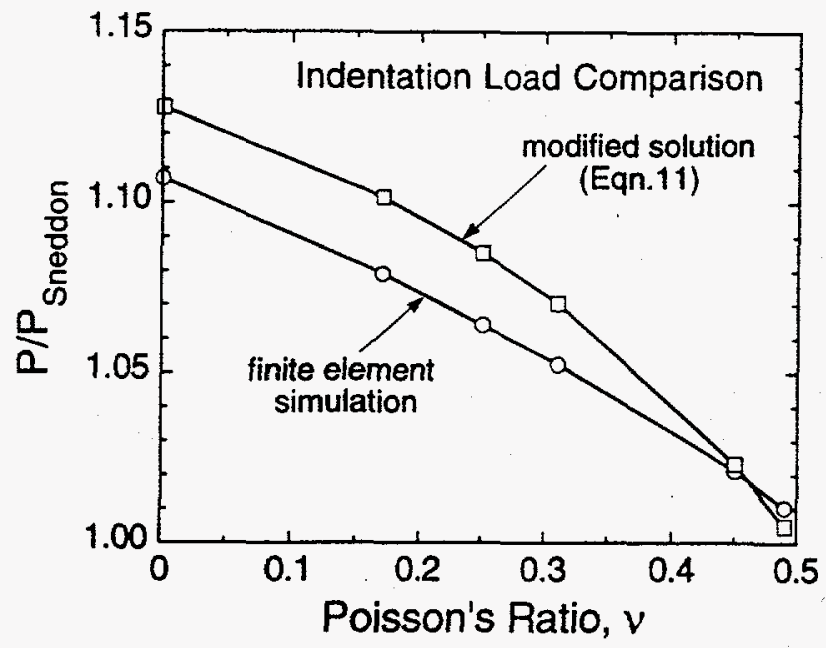

Fig. 2. A comparison of the indentation loads observed in the finite element calculations to Sneddon's solution and the approximate modified solution. Loads are normalized with respect to Sneddon's results and plotted as a function of Poisson's ratio.

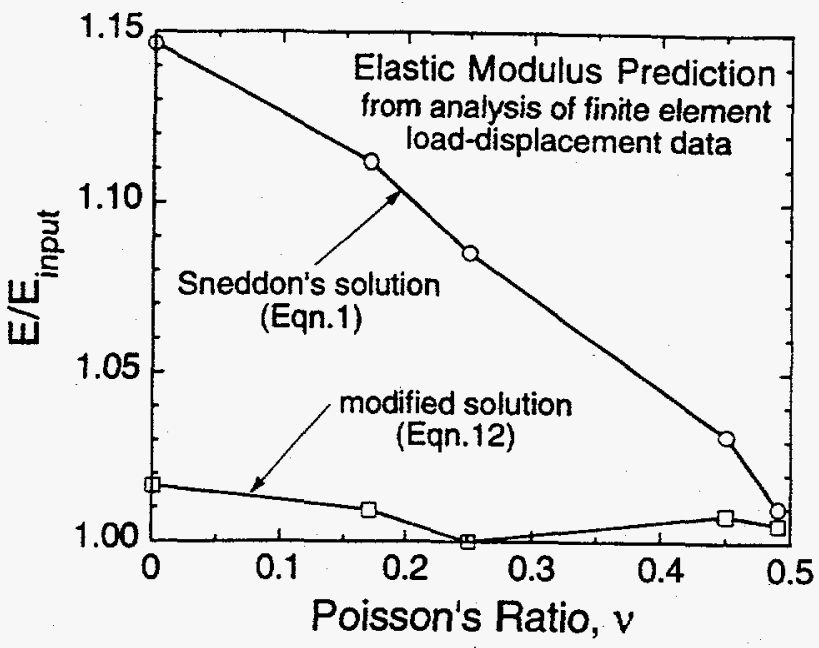

Fig. 3. A comparison of the effective elastic moduli derived by applying Eqns. 1 and 12 to the finite element load-displacement data. The moduli are normalized with respect to the effective modulus input into the simulations and plotted as a function of Poisson's ratio.

The simulations were conducted for various $v^{\prime} s$ and $E$ 's but with the effective modulus $E_{\text {eff }}=$ $E /\left(1-v^{2}\right)$ held constant at $E_{\text {eff }}=90.85 \mathrm{GPa}$. The reason for performing the simulations in this way was to explore an important difference between the dependencies on Poisson's ratio of Sneddon's original solution and the modified solution derived here. The difference may be seen by comparing Eqns. 1 and 12, which shows that $E_{\text {eff }}$ computed from Sneddon's solution should be independent of Poisson's ratio, while $E_{\text {eff }}$ derived from the modified solution is not because $\beta$ is a function of $v$. The same is true of the indentation load at a fixed depth.

Figures 2 and 3 present a comparison of the finite element results with the two solutions. Figure 2 shows the dependence of the predicted indentation loads on Poisson's ratio (the loads are normalized with respect to those predicted by Sneddon's solution), while in Figure 3, the variation of the elastic moduli predicted by Eqns. 1 and 12 are compared. In computing the elastic moduli, the contact areas were determined directly by examination of the finite element mesh rather than indirectly through an analysis of the load-displacement data, as would be done in nanoindentation experiments.

The results in Figure 2 show that the indentation loads in the finite element simulations are larger than those predicted in Sneddon's solution. For $v=0$, the error in Sneddon's solution according to the finite element calculations is about $11 \%$, but the error decreases essentially to zero at $v=0.5$, consistent with the fact that the radial displacements of the surface vanish as $v$ approaches 0.5 . On the other hand, the modified solution matches the finite element results reasonably well, thereby providing justification for the assumptions made in deriving it, as well as demonstrating its potential for application in nanoindentation analysis procedures. Figure 3 demonstrates the implications for Young's modulus measurement from nanoindentation loaddisplacement data. It shows that if Sneddon's results are used (Eqn. 1), errors as large as 14\% may be obtained, but these errors can be largely avoided by using the modified solution (Eqn. 12). The results emphasize the need for modification of Eqn. 1 in the manner outlined here if accurate elastic moduli are to be derived from analyses of nanoindentation data.

\section{CONCLUSIONS AND IMPLICATIONS FOR NANOINDENTATION DATA ANALYSIS}

The analyses and finite element simulations presented in this paper show that corrections to Sneddon's solution for indentation of an elastic half-space by an axisymmetric indenter are 
needed if accurate measurements of elastic modulus are to be obtained in nanoindentation experiments with sharp indenters. Failure to make the corrections results in an underestimation of the indentation load and contact stiffness and an overestimation of the elastic modulus by as much as $13 \%$. The amount of the correction depends on Poisson's ratio and can be estimated using procedures developed in the paper.

It is important to note that the contact area deduced from Sneddon's solution may also be affected by the problems identified here. Corrections to the contact area, if required, would have implications not only for the measurement of elastic modulus, but for the measurement of hardness as well. It is also important to note that the analyses presented in this paper apply strictly only to elastic deformation. For sharp contact involving both elastic and plastic deformation, the situation is considerably more complex. We are currently studying the role that these issues play in modifying the results [9].

\section{ACKNOWLEDGMENTS}

This research was sponsored by the Division of Materials Sciences, U.S. Department of Energy, under contract DE-AC05-960R22464 with Lockheed Martin Energy Research Corp. One of the authors (GMP) is grateful for sabbatical support provided by the Oak Ridge National Laboratory.

\section{REFERENCES}

1. I.N. Sneddon, Int. J. Engng. Sci. 3, 47 (1965).

2. I.N. Sneddon, Fourier Transforms (McGraw-Hill Book Company, Inc., 1951), pp. 450-467.

3. G.M. Pharr, W.C. Oliver, and F.R. Brotzen, J. Mater. Res. 7, 613 (1992).

4. W.C. Oliver and G.M. Pharr, J. Mater. Res. 7, 1564 (1992).

5. M.F Doerner and W.D. Nix, J. Mater. Res. 1, 601 (1986).

6. A. Bolshakov, W.C. Oliver, and G.M. Pharr, J. Mater. Res. 11, 760 (1996).

7. J.E. Ritter, T.J. Lardner, D.T. Madsen, and R.J. Gionazzo, in Materials in Microelectronic and Optoelectronic Packaging, American Ceramic Society, p. 379.

8. T.A. Larsen and J.C. Simo, J. Mater. Res. 7, 618 (1992).

9. A. Bolshakov and G.M. Pharr, unpublished work.

\section{DISCLAIMER}

This report was prepared as an account of work sponsored by an agency of the United States Government. Neither the United States Government nor any agency thereof, nor any of their employees, makes any warranty, express or implied, or assumes any legal liability or responsibility for the accuracy, completeness, or usefulness of any information, apparatus, product, or process disclosed, or represents that its use would not infringe privately owned rights. Reference herein to any specific commercial product, process, or service by trade name, trademark, manufacturer, or otherwise does not necessarily constitute or imply its endorsement, recommendation, or favoring by the United States Government or any agency thereof. The views and opinions of authors expressed herein do not necessarily state or reflect those of the United States Government or any agency thereof. 